\title{
A WEAK ESTIMATE OF THE FRACTAL DIMENSION OF THE MANDELBROT BOUNDARY
}

\author{
Bruce ELENBOGEN and Thomas KAEDING \\ Mathematics Department. The Lniversity of Michigan-Dearborn, Dearborn, MI 43128-1491, USA
}

Received 6 September 1988; revised manuscript received 18 January 1989; accepted for publication 29 January 1989

Communicated by D.D. Holm

\begin{abstract}
A technique to compute fractal dimension as defined by the Kolmogorov capacity is discussed. The method is used to compute fractal dimension for several standard curves and the boundary of the Mandelbrot set. This estimate of fractal dimension, although very rough, refutes Milnor's conjecture that the Hausdorff dimension of the Mandelbrot boundary is 2.
\end{abstract}

\section{Introduction}

The fractal dimension of an object is currently being utilized to explore a variety of physical phenomena [1]. Measurements of fractal dimension have been made to gain insights into molecular aggregation, galaxies, protein behaviour, and geological formations $[2,3]$. The need to estimate dimension numerically has also arisen in several problems connected with turbulence theory, the analysis of stochasticity in dynamical systems, and in combustion theory [4]. One definition of fractal dimension is given by the Kolmogorov capacity [5]. The capacity is an upper bound to the Hausdorff dimension for all objects and identical to the Hausdorff dimension for a wide class of objects [6].

One important and highly complex theoretical fractal set is the boundary of the Mandelbrot set. The Mandelbrot set classifies chaotic behaviour for all complex quadratic functions [7]. Unfortunately, it is not a strange attractor and thus not amenable to more efficient methods of determining fractal dimension [8]. Further, at present, the Hausdorff dimension of the Mandelbrot boundary $(\partial \mathrm{M})$ has eluded analytical determination. This paper investigates a method of numerically estimating fractal dimension which is used to give a weak upper bound for the Hausdorff dimension of the Mandelbrot boundary.

\section{The method}

We begin by stating the definition of Kolmogorov capacity $d_{\mathrm{c}}$ as given in ref. [5],

$d_{\mathrm{c}}=\lim _{\epsilon, 0} \log N(\epsilon) / \log (1 / \epsilon)$,

where if the set in question is a bounded subset of a $p$-dimensional Euclidean space $\mathbb{R}^{\prime \prime}$, then $N(\epsilon)$ is the minimum number of $p$-dimensional cubes of side $\epsilon$ needed to cover the set.

The numerical methods used in the literature to estimate capacity require covering of the object of interest with balls of various radii. The dimension is then computed by measuring the slope of the log-log graph of number of cover elements versus the radius of the cover elements.

The main difference in these methods lies in the method of determining the cover. A standard approach to forming a cover of a closed curve is to "walk" along the curve with uniformly sized steps. This has been used to find the fractal dimension of coastlines and particles in nature [9-11]. By walking the curve with uniform covers, the number of cover elements needed to complete the cover is close to the minimum number. An initial attempt to utilize this method in investigation of the Mandelbrot boundary has shown to be impractical. The branching and complexity of $\partial \mathrm{M}$ is such that computer algorithms attempting to traverse this curve with no 
memory of past steps turn back on themselves and wander forever. Adding memory of all past steps involves the building of a data file of boundary points, which, again due to the complexity of the Mandelbrot boundary, would prove to be uninformative on large scales of length and unreasonably time-consuming on small scales of length.

Another method of estimating capacity is to use a box-counting method. The object of study is placed on a grid of just-touching square boxes of side length $2^{-1}$. Let $N(n)$ denote the number of boxes of side length $2^{-n}$ which intersect the object. It can be shown [6] that the capacity $d_{c}$ is also given by

$d_{c}=\lim _{n \rightarrow \infty} \log N(n) / \log 2^{n}$.

Chorin has used this method for the estimation of zeros of Brownian motion [4], and Clarke has applied this method to surfaces [3]. In contrast to their studies, however, the Mandelbrot boundary is much more difficult to locate. Consequently, the inaccuracy in determination of the set allows some boxes (cover elements) to escape detection. Using Chorin and Clarke's method of cover construction, smaller cover elements embedded in inaccurate larger cover elements are also lost from consideration. The method utilized in this study is a modification of their technique.

The difference between Chorin's method and the method discussed in this paper lies in the manner of generating cover sets. Rather than beginning with a largest initial cover, we begin with the cover of finest resolution. From this cover, larger covers are more easily formed. Further, subsequent covers are at least as accurate as their predecessors. Potential cover elements are formed by dividing the plane into a uniform grid of squares. A cover consists of a set of all squares which contain both interior and exterior points of the object of study.

The connectedness of an object's boundary is used to reduce the number of points that need to be evaluated for membership in the object. Douady [12] showed that the Mandelbrot set is connected, and hence is appropriate for this method of analysis. The connectedness of a boundary implies that cover membership can be determined solely by checking the perimeter of a square cover element for bound- ary points. This reduces the number of computations from order $n^{2}$ to order $n$.

The method begins with a list of sides of squares known to contain boundary points. Each element of the list is sequentially examined to determine if any of the six surrounding square segments intersects the boundary. Sides of squares that contain boundary points are added to the list. Segments in the list that fail to contain boundary points are removed from further consideration. In this manner a minimum number of points are analyzed. From this list of square sides, it is easy to obtain a list of square cover elements and generate the double-log plot whose slope estimates the fractal dimension.

\section{Results}

The method was applied to a segment of the line $y=x$, the unit circle, the Koch snowflake [10], a space-filling curve similar to the Peano curve, and the boundary of the Mandelbrot set. All figures were restricted to lie in the rectangle $-2 \leqslant x \leqslant 2$, $-2 \leqslant y \leqslant 2$, with the reflective symmetry of the figures with respect to the $x$-axis (real axis) exploited to ignore points below the $x$-axis. Experiments were performed with the length of a square's side at $2^{-8}$, $2^{-9}, 2^{-10}$, and $2^{-11}$. The use of lengths that are reciprocals of powers of two allowed the use of cover elements that are represented by integers. This made computations simpler and more accurate, and allowed the use of Richardson extrapolation [13]. The side of cover square was considered not to contain a boundary point if after checking 17 equally spaced points on that segment, no two points were found on different sides of the boundary. Subsequent covers had sides that ranged in length from twice to eighteen times the length of the original cover size. Least squares analysis was used to determine lower bounds for the dimension as the absolute value of the slope of the $\log -\log$ plot of cover number versus cover size. This sequence of values was then accelerated using Richardson extrapolation to compute a best estimate for the fractal dimension.

Although determination of the boundary of line segments and circles is straightforward, the Koch snowflake, the space-filling curve, and the Mandelbrot set require iterative schemes. A point is consid- 
ered outside the boundary of the snowflake if it is exterior to the original triangle defining the zeroth iteration of the snowflake and is exterior to that triangle after 32 appropriate translations and rotations. The computed Koch snowflake is shown in fig. 1. The space-filling curve is approximated by a grid of $3^{64}$ lines in both the $x$ and $y$ directions restricted to the unit square (see fig. 2 ). A point $\mu$ is considered outside the boundary of the Mandelbrot set if the forward orbit of 0 has complex modulus greater than 2 after 256 iterations of the map $f_{\mu}(z)=z^{2}-\mu$. The

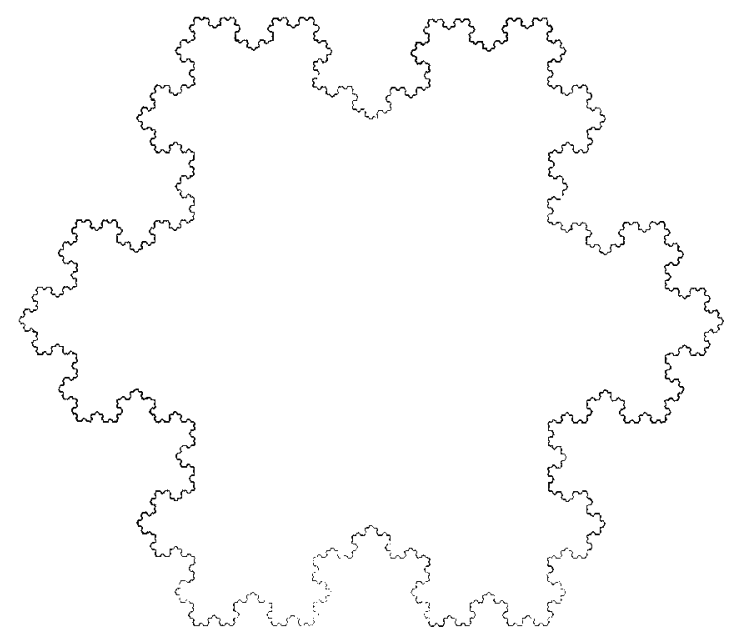

Fig. I. Approximation to Koch snowflake.

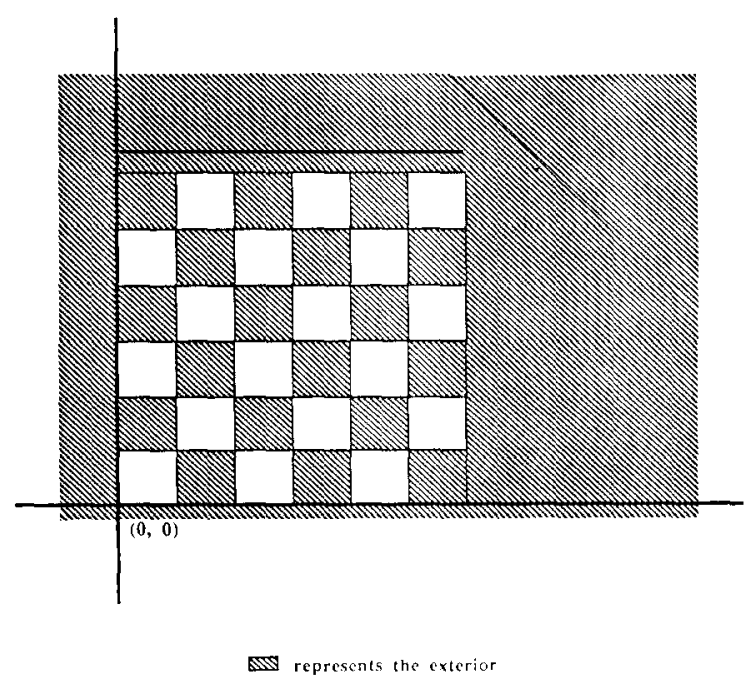

Fig. 2. Enlargement of space-filling curve.

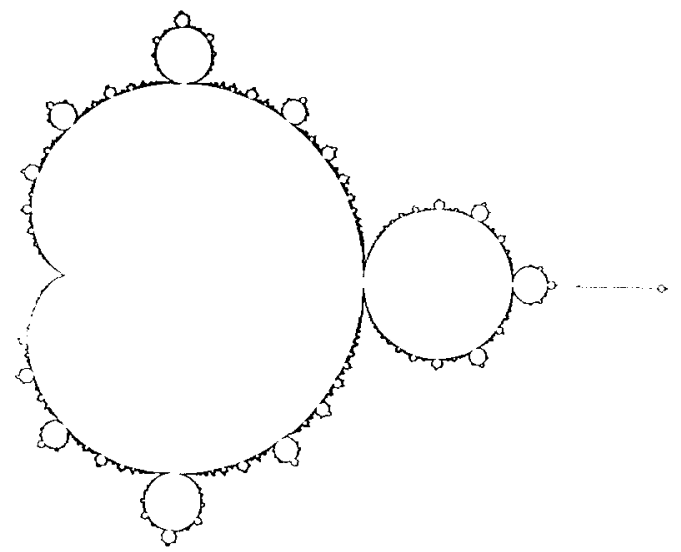

Fig. 3. Approximation to Mandelbrot boundary.

computed approximation to the Mandelbrot Boundary is shown in fig. 3 .

\section{Analysis and error}

In order to verify the method, the program was tested on several curves whose capacities equal their Hausdorff dimensions and are known exactly. The estimates for the capacity of these curves are presented in table 1, along with an estimate for the capacity for the Mandelbrot boundary.

The program used was run on a 6 mips Vax in double precision FORTRAN. The finest cover of $\partial \mathrm{M}$ required approximately 10 hours of cpu time and 800 kilobytes of main memory. The double-log plots of data with finest resolution appears in fig. 4.

In general, smaller cover sizes produce better estimates of capacity. Hence, estimates for each curve are monotonically increasing with $n$. As $n$ is increased, the estimates of $d_{\mathrm{c}}$ approach the true value from below. Computer roundoff error enters the computation only in the determination of the segments that intersect an object's boundary. Once boundary segments are determined, the remainder of the calculation involve only integers and are exact.

Further, due to the inherent drawbacks in the computational method, the results obtained for the Mandelbrot boundary should be taken as a lower bound, and not as estimates of the capacity itself. We make this assertion for three reasons: first, a computer cannot check all points on the perimeter of a 
Table 1

\begin{tabular}{|c|c|c|c|c|c|c|}
\hline Curve & $\begin{array}{l}\text { Capacity } \\
\text { for } t=2^{-8}\end{array}$ & $\begin{array}{l}\text { Capacity } \\
\text { for } \epsilon=2^{-9}\end{array}$ & $\begin{array}{l}\text { Capacity } \\
\text { for } \epsilon=2^{-10}\end{array}$ & $\begin{array}{l}\text { Capacity } \\
\text { for } \epsilon=2^{-11}\end{array}$ & $\begin{array}{l}\text { Estimated } \\
\text { capacity }\end{array}$ & $\begin{array}{l}\text { Theoretical } \\
\text { capacity }\end{array}$ \\
\hline line segment & 0.99 & 0.99 & 0.997 & 0.999 & $1.000 \pm 0.0003$ & 1 \\
\hline unit circle & 0.95 & 0.965 & 0.995 & 0.997 & $0.997 \pm 0.01$ & 1 \\
\hline Koch snowflake & 1.24 & 1.25 & 1.27 & 1.26 & $1.26 \pm 0.02$ & 1.2619 \\
\hline Peano curve & 2.0 & $\mathrm{NA}$ & NA & NA & $2.0 \pm 0.01$ & 2 \\
\hline$\partial \mathrm{M}$ & 1.29 & 1.42 & 1.52 & 1.61 & $1.64 \pm 0.1$ & unknown \\
\hline
\end{tabular}

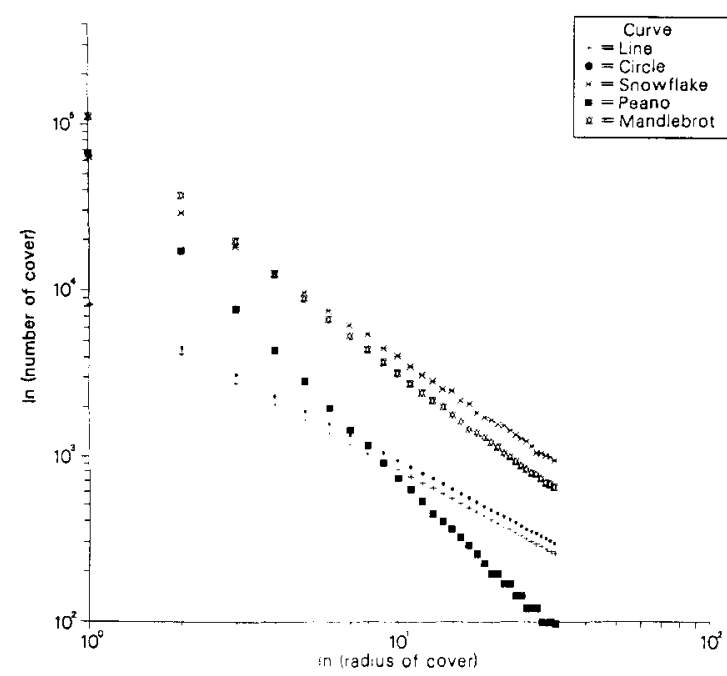

Fig. 4. Double-log plot of cover data at finest resolution.

cover element for membership in the Mandelbrot set, and hence very fine features of $\partial \mathrm{M}$ will be overlooked. In fact, at the resolution of $2^{-11}$, many of the finer filaments of $\partial \mathrm{M}$ were not found. This would tend to decrease the number of smaller covers, while scarcely affecting the number of larger covers. This disproportional change in the number of cover elements decreases the absolute slope of the log-log graph of cover elements to cover size, and thus lowers the estimate of the capacity. Second, the iteration process used to determine membership in the Mandelbrot set allows nearby exterior points to be considered part of the set. This tends to thicken the set and obscure some of the more delicate features, which would result in lowering the estimate for the capacity. Lastly, the definition of Kolmogorov capacity applies in the limit as cover size tends to zero. The detail of the Mandelbrot boundary becomes more complex and intricate as one examines it on smaller and smaller scales.
Numerical errors were introduced into the computation from two sources: the determination of the boundary and determination of slope of the curve. The error due to determination of the boundary was assumed to be normally distributed, so that a least squares determination of the slope provided the best estimate. However, the statistical error bounds do not take into account that the slopes are part of a limit process. It was therefore decided to use the error bounds predicted by the Richardson extrapolation method. This error proved to be accurate for all base curves examined.

\section{Conclusions}

It appears that the method yields a weak estimate of the fractal dimension of objects in a reasonable amount of time. The simplicity of the method allows it to be used to measure the fractal dimensions of objects that are not strange attractors. As finer covers are used, the results for the fractal dimensions monotonically approach the true value from below. Our estimate for the fractal dimension of the Mandelbrot boundary is $d_{\mathrm{c}}(\partial \mathrm{M})=1.64 \pm 0.1$. This result lends evidence that refutes Milnor's conjecture that the Hausdorff dimension of $\partial \mathrm{M}$ is 2 [14].

This method for computation of fractal dimension can also be applied to the boundaries of any sets that have connected exteriors and interiors with non-zero measure. This extension can include application to high dimensions such as fractal surfaces as investigated by Clarke [3]. However, such an extension would be increasingly time-consuming with higher dimensions and may prove impractical. The method can also be modified to test the sensitivity of the estimate to the shape of cover elements. 


\section{Acknowledgement}

This work was partially supported by a grant from The University of Michigan-Dearborn. Also we wish to thank L.P. Johnston for the expert technical support, and the referees for their extensive and insightful comments.

\section{References}

[1] M. La Brecque, Mosaic 14 (1986) 34.

[2] H.E. Stanley and N. Ostrowsky, On growth and form (fractal and non-fractal patterns in physics (Nijhoff, The Hague, 1985).

[3] K.C. Clarke, Comput. Geosci. 12 (1986) 713.

[4] A.J. Chorin, J. Comput. Phys. 46 (1982) 390.

[5] A.N. Kolmogorov, Dokl. Akad. Nauk SSSR 119 (1958) 861.

[6] J.D. Farmer, E. Ott and J.A. Yorke, Physica D 7 (1983) 153.
[7] P. Blanchard, Bull. (New Series) Am. Math. Soc. 11 (1984) 85.

[8] P. Grassberger and I. Procaccia, Physica D 9 ( 1983 ) 189.

[9] B.B. Mandelbrot, The fractal geometry of nature (Freeman. San Francisco, 1982).

[10] B.B. Mandelbrot, Fractals: form, chance, and dimension (Freeman, San Francisco, 1977).

[11] S.K. Kennedy and W.H. Lin, Comput. Geosci. 12 (1986) 705.

[12] A. Douady, Asterisque 105 (1983) 39.

[13] F.B. Hildebrand, Introduction to numerical analysis (McGraw-Hill, New York. 1974).

[14] J. Milnor, Self-similarity and hairiness in the Mandelbrot set, preprint, Institute for Advanced Study.

[15] R.L. Devaney, An introduction to chaotic dynamical systems (Benjamin/Cummings, Menlo Park, 1986).

[16] H.O. Peitgen and P.H. Richter, The beauty of fractals (Springer, Berlin, 1986).

[17] B.B. Mandelbrot, Ann. N.Y. Acad. Sci. 357 (1980) 249.

[18] K.J. Falconer. The geometry of fractal sets (Cambridge Univ. Press, Cambridge, 1985).

[19] P. Fatou, Acta Math. 47 (1926) 337. 